\title{
Dynamic Medical Material Distribution Model Based on Epidemic Diffusion Rule and Cluster- ing Approach
}

\author{
Guangmin $\mathrm{Wu}^{1 \mathrm{a}}$, Jiarun Peng ${ }^{2}$, and Bowen $\mathrm{Xi}^{1}$ \\ ${ }^{1}$ Department of Electric Power Engineering, North China Electric Power University, 102206 Baoding \\ Hebei, China \\ ${ }^{2}$ Department of Mathematics and Physics, North China Electric Power University, 102206 Baoding \\ Hebei, China
}

\begin{abstract}
Due to the fact that the dynamic medical material distribution is vital to the quick response to urgent demand when the Ebola virus occurs, the optimal distribution approach is explored according to the Ebola virus diffusion rule and different severity of the epidemic. First, we choose the more serious epidemic state of Sierra Leone in West Africa as the research object and the SIQR (susceptible, infected, quarantined, required) epidemic model with pulse vaccination is introduced to describe the Ebola diffusion rule and obtain the demanded vaccine and drug in each pulse. Based on the SIQR model, thirteen areas in Sierra Leone are classified into three emergency levels by clustering analysis. Then a dynamic medical material distribution model is formulated, with goals of both reducing the transportation cost and shortages. The results indicate that the proposed approach can make an outstanding contribution to fight against the Ebola virus.
\end{abstract}

Keywords. the Ebola virus; SIQR model; clustering analysis; medical material distribution model

\section{Introduction}

The deadly hemorrhagic fever Ebola was firstly discovered in 1976, and it has haunted in people's mind for twenty years. Since December 2013, an ongoing outbreak of Ebola in West Africa has infected at least 20,000 people in Guinea, Sierra Leone and Liberia, including 8,000 who died, according to the World Health Organization.

Nowadays, the world medical association has announced that their new medication could stop Ebola and cure patients whose illness condition is not worsened. However, how to eliminate or fight against the Ebola virus is still a great challenge. The difficulty mainly lies in

\footnotetext{
${ }^{a}$ Corresponding author:695746712@qq.com
} 
two aspects: how to describe the spread characteristics of the Ebola virus to predict the required number of vaccines or drugs? How to establish the possible transportation system for reasonable distribution of vaccines or drugs? In addition, the speed to manufacture a vaccine or drug is also an important factor to be considered.

Besides, from the domestic and foreign literature, we can see that the majority of emergency material distribution model can only solve the resource allocation problem of single point of disaster rescue ${ }^{[1]}$. But due to the completely different severity of the epidemic, the outbreak of the Ebola virus in the West African countries and the emergency degrees of each epidemic are not the same. When the affected sites arise simultaneously, the competitive relationship between drug resource demands will emerge, so the accumulation of each single affected site's optimal solution will not be able to reach the optimum on the whole. Then the severity of the epidemic should be taken into consideration as important factors on the distribution of materials.

Therefore, according to the Ebola virus spreading rules and different severity of epidemic, how to distribute drugs reasonably in the affected areas with the limited resources and effectively fight against the Ebola virus will be the focus of this paper.

\section{SIQR epidemic diffusion model}

To facilitate the progress of model formulation, we make the following two assumptions. 1) The total number of $\mathrm{N}$ stays unchanged. The crowd is classified into susceptible people, infected people, quarantined people and recovered people. 2) Affected areas are isolated from each other without population mobility when epidemics hit.

Due to the great difficulty in eradicating the epidemic by a one-time vaccination, we use the pulse vaccination method with a low vaccination rate to control the epidemic. Thus, the SIQR epidemic model with pulse vaccination being close to the actual situation is employed to obtain the amount of vaccine demanded. The SIQR epidemic model with pulse vaccination is adopted as Figure $1^{[2]}$.

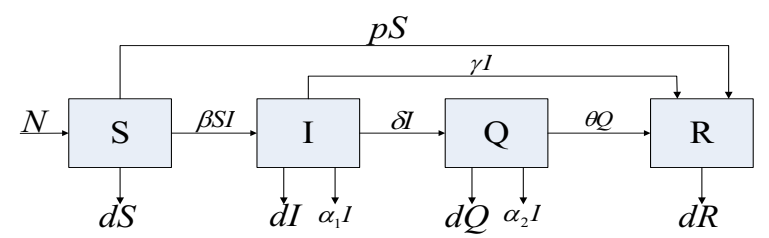

Figure 1. SIQR model with pulse vaccination

$$
\begin{aligned}
& \left\{\begin{array}{l}
\frac{d S}{d t}=[d-(\beta I(t)+d)] S(t) \\
\frac{d I}{d t}=\beta S(t) I(t)-\left(\delta+\gamma+d+\alpha_{1}\right) I(t) \\
\frac{d Q}{d t}=\delta I(t)-\left(d+\alpha_{2}+\theta\right) R(t) \\
\frac{d R}{d t}=\gamma I(t)+\theta Q(t)-d R(t) \\
t \neq k \tau, k=0,1,2,3 \ldots
\end{array}\right.
\end{aligned}
$$

Where, $S\left(k \tau^{+}\right)=\lim S(k \tau+\lambda)$

$$
R\left(k \tau^{+}\right)=\lim _{\lambda \rightarrow 0^{+}} R(k \tau+\lambda)
$$


As mentioned above, $I$ stands for the number of patients who are already infected and show symptoms, and the initial values of $S, I$ and $R$ are given. We can get the value of $I$ at some moment by solving differential equations (1). We expect that $I$ can stay in a lower level in this model, which means the spreading of the infectious disease is under effective control. We can see from the infectious disease model, in order to carry out the effective control of $I$, we should decrease the propagate coefficient of the disease and simultaneously improve the recovery rate of infectious diseases. From the perspective of distribution of vaccines or drugs, we should ensure that there is sufficient rescue drug for emergency treatment of patients to increase the probability of recovery, thus to effectively control the increase of the number.

Assuming that $I(t)$ is a function decreasing with time, then the infected population will eventually tend to 0 . Under the condition that $\frac{d I}{d t}<0$, we can get the minimum value for the vaccination rate of $\mathrm{P}^{[3]}$ :

$$
\begin{aligned}
p=\min \left\{\frac{d T\left(e^{d T}-1\right)\left(\beta-\delta-\gamma-d-\alpha_{1}\right)}{\beta\left(e^{d T}-1\right)-d T\left(\beta-\delta-\gamma-d-\alpha_{1}\right)},\right. \\
\left.\frac{\left(e^{d T}-1\right)\left(\beta-\delta-\gamma-\alpha_{1}\right)}{\delta+\gamma+\alpha_{1}}\right\}
\end{aligned}
$$

The forecast of the demand changing over time is as follows:

$$
D_{j}^{k}(t)=p^{k} S_{j}\left(t^{-}\right)
$$

The model parameters are given as Table 1.

Table 1. Parameters of SIQR

\begin{tabular}{llcc}
\hline$d$ & $\beta$ & $\delta$ & $\gamma$ \\
\hline 0.00001 & 0.0005 & 0.3 & 0.002 \\
$\alpha_{1}$ & $\alpha_{2}$ & $\theta$ & $p$ \\
0.0001 & 0.00005 & 0.3 & 0.15 \\
\hline
\end{tabular}

We choose 13 cities in Sierra Leone as the epidemic areas, assuming impulsive vaccination cycle of $\mathrm{T}=5$, using the numerical ODE45 function of MATLAB (which can be infectious disease model solution). Due to limited space, we only list the numerical solution of four cities, as shown in Figure 2.

As shown in Figure 2, most infected areas achieve stability within 5 to 10 vaccination cycles.

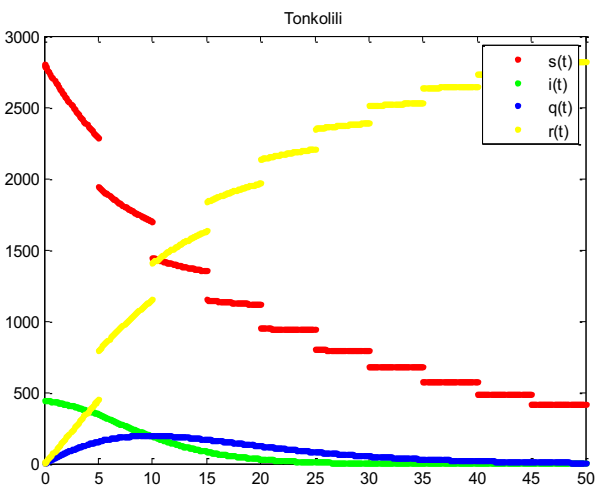

(a)

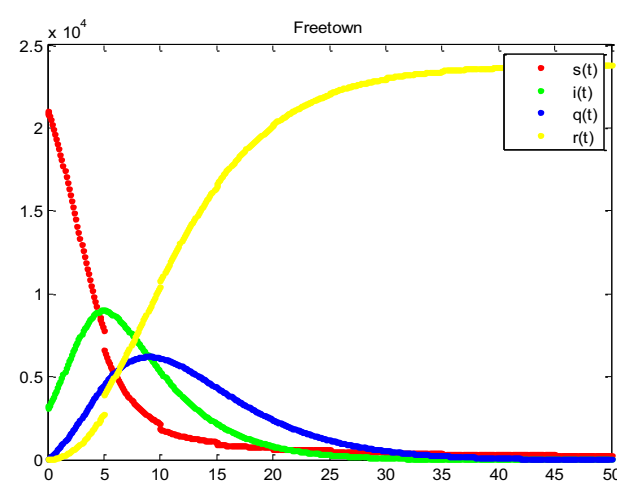

(b) 


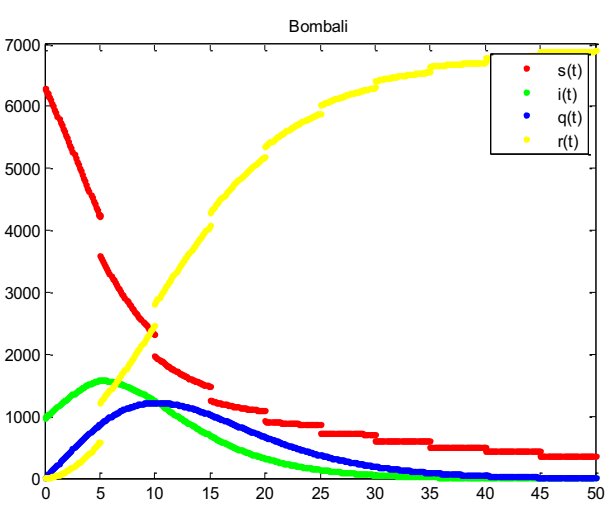

(c)

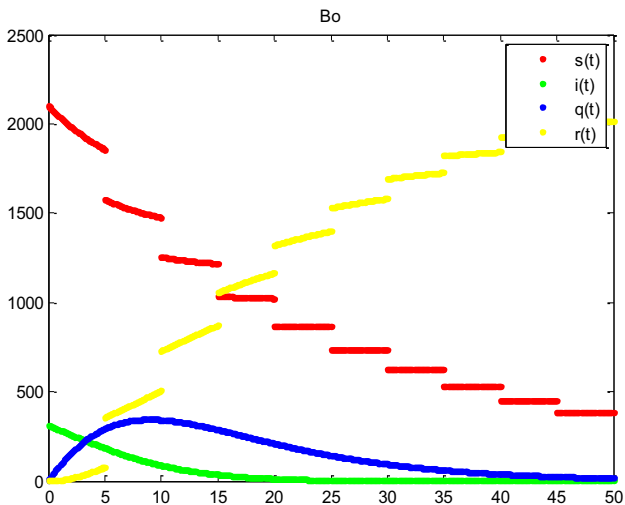

(d)

Figure 2. The four cities in the process of injection vaccine in 50 days.

\section{Dynamic medical material delivery model}

\subsection{Clustering in affected areas}

Because of differences in the serious degree of time and the outbreak time, in this section we will use clustering to the affected areas, and divide the emergency degree of epidemic areas into different levels in order to achieve more effective control of the epidemic spreading.

The following 3 indexes are selected as clustering basis.

Mortality $w_{j}^{1}$ : the proportion of deaths in patients infected with Ebola virus in epidemic area $j$.

Morbidity rate $w_{j}^{2}$ : the proportion of those infected with Ebola virus in the total population in epidemic area $j$.

The number of cases $w_{j}^{3}$ : the total number of people infected with Ebola virus in epidemic area $j$.

Through online information access ${ }^{[4]}$, we obtained the following processed data:

Table 2. Data of infected areas

\begin{tabular}{lllll}
\hline Number & Infected Areas & The number of cases & Morbidity rate & Mortality \\
\hline 1 & Bo & 314 & 0.00056 & 0.3445 \\
2 & Bombali & 979 & 0.00225 & 0.2611 \\
3 & Bonthe & 5 & 0.00004 & 0.5833 \\
4 & Kailahun & 565 & 0.00138 & 0.3209 \\
5 & Kambia & 141 & 0.00045 & 0.3870 \\
6 & Kenema & 498 & 0.00091 & 0.3499 \\
7 & Koinadugu & 103 & 0.00041 & 0.4492 \\
8 & Kono & 235 & 0.00067 & 0.4036 \\
9 & Moyamba & 202 & 0.00080 & 0.3355 \\
10 & Port & 1,297 & 0.00259 & 0.2701 \\
11 & Pujehun & 31 & 0.00012 & 0.4038 \\
12 & Tonkolili & 445 & 0.00115 & 0.2811 \\
13 & Freetown & 3,106 & 0.00187 & 0.2449 \\
\hline
\end{tabular}


Clustering analysis steps are as follows:

Step 1 Standard processing. In order to eliminate the influence of different magnitude and dimension brought, we need data standardization:

$$
w_{j}^{\prime}=\frac{w_{j}-w_{j \min }}{w_{j \max }-w_{j \min }}
$$

Step 2 The definition of the distance function. The distance function is defined as a measure of the degree of similarity between data points, and the measurement of similarity between different kinds of data in the same feature space is very important for clustering. We choose the Euclidean distance as the distance function, it is defined as follows:

$$
d(x, y)=\left(\sum_{k=1}^{p}\left|x_{k}-y_{k}\right|^{2}\right)^{\frac{1}{2}}
$$

Step 3 Model-based clustering. It is a very important step that the data objects are divided into different classes. Common clustering methods include model-based clustering, densitybased clustering, and clustering based on grid and so on. In this paper, we use the model-based clustering.

From the above, we can divide 13 epidemic areas into 3 categories. The twice clustering results are now tabulated as follows, and corresponding levels of epidemic weight are given.

Table 3. Results of clustering in affected areas

\begin{tabular}{llll}
\hline & The first clustering & The second clustering & Weight \\
\hline Group-1 & $2,10,13$ & $2,10,13$ & 0.6 \\
Group-2 & $4,6,12$ & $1,4,6,12$ & 0.4 \\
Group-3 & $1,3,5,7,8,9,11$ & $3,5,7,8,9,11$ & 0.2 \\
\hline
\end{tabular}

The size of the weight reflects the development trend of the epidemic, the greater the weight is, the more serious the epidemic is, and otherwise it is not serious.

The size of the weight reflects the development trend of the epidemic, the greater the weight is, the more serious the epidemic is, and otherwise it is not serious. In order to illustrate the clustering result figuratively, we will mark the cities of Sierra Leone with different colors, as shown in the figure. The darker colored places demonstrate that the epidemic is more serious.

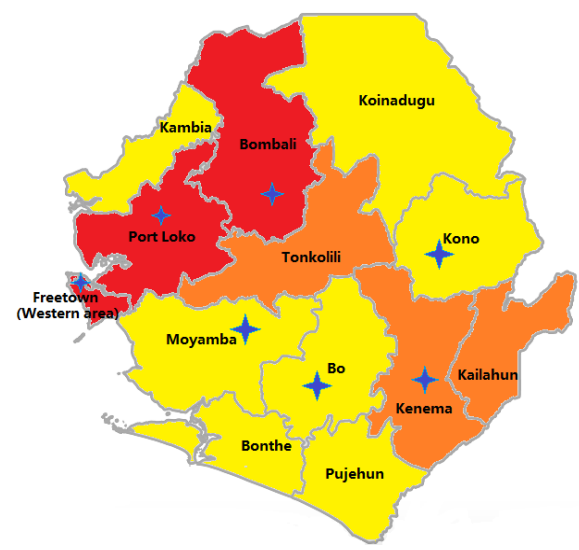

(a)

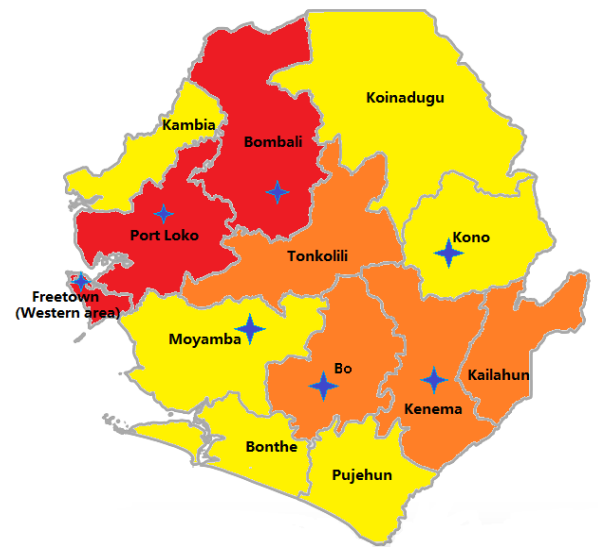

(b)

Figure 3. Epidemic distribution of Sierra Leone. 
From the above chart, we can see that the epidemic is mainly concentrated in northwest Sierra Leone, which is consistent with the distribution we have known ${ }^{[5]}$. The blue marks stand for 7 distribution stations, which we choose according to the epidemic distribution and the actual location of distribution stations.

\subsection{Cluster-based medical material delivery model}

\section{Objective1: minimize transportation costs}

Considering that the cost of transportation is not only related with the transport cost per unit of different modes, it is also related to the number of the transport and the distance. We can find the transport distance through using data $t$. At the same time, in order to simplify the model, we can set the transport cost as a specific constant.

Take the vaccine as an example, and the minimum transportation cost for any reserve is to send vaccine to any epidemic area $j$ for $\mathrm{k}$ times. It can be described as follows:

$$
\min F_{1}^{1 k}(t)=\sum_{\forall k} \sum_{\forall j} \sum_{i=1}^{n} C_{i j} X_{i j}^{v k}(t) L_{i j}
$$

We can get the following results by normalizing the data:

$$
\min \overline{F_{j}^{1 k}}(t)=\frac{F_{j}^{1 k}(t)-F_{j}^{1 k}(t)_{\min }}{F_{j}^{1 k}(t)_{\max }-F_{j}^{1 k}(t)_{\min }} \forall(k, j), t=k \tau
$$

\section{Objective 2: minimize the amount of shortages}

By the model II, we can predict the demand quantity of vaccines or drugs from the epidemic areas, and we should try to meet the demand of various epidemic areas in actual distribution, so that the deviation between the actual transport and forecasting demand can be minimum.

Take the vaccine as an example, the minimum number out of stock can be described as follows ${ }^{[3]}$ :

$$
\min F_{j}^{2 k}(\mathrm{t})=\sum_{\forall \mathrm{k}} \sum_{\forall j} D M_{j}^{v k}(t)-\sum_{\forall k} \sum_{\forall j} \sum_{i=1}^{n} X_{i j}{ }^{v k}(t)
$$

Where, $X_{i j}^{v k}(t)$ is the actual number of vaccine delivery from $\mathrm{i}$ to $\mathrm{j}$ in the first $\mathrm{k}$ transportation.

We can get the following results by normalizing the data:

$$
\min \overline{F_{j}^{2 k}}(t)=\frac{F_{j}^{2 k}(t)-F_{j}^{2 k}(t)_{\min }}{F_{j}^{2 k}(t)_{\max }-F_{j}^{2 k}(t)_{\min }} \forall(k, j), t=k \tau
$$

The fact is that the two objectives are with different priorities. Weight coefficients $\mu_{1}$ and $\mu_{2}$ are introduced to integrate the two objective functions into a composite optimization problem. We transfer the multi-objective optimization problem into a single objective optimization problem of minimizing the total cost.

Thus the composite objective function $F_{j}^{k}(t)$ is as follows:

$$
\min F_{j}^{k}(t)=\mu_{1} \overline{F_{j}^{2 k}(t)}+\mu_{2} \overline{F_{j}^{1 k}(t)} \forall(k, j), t=k \tau
$$

On the basis of the above objective functions (9) and according to the severity of different epidemic areas, we design the objective function of a plurality of epidemic areas as follows: 


$$
\min F^{k}(t)=\sum_{j=1}^{m}\left(1-\omega_{j}^{k}(t)\right) F_{j}^{k}(t) \forall \boldsymbol{k}, t=k \tau
$$

Where: $\omega_{j}^{k}(t)$ stands for the weight of the epidemic area.

\section{Constraint 1: demand constraints}

The total amount of demand vaccine and medicine for the affected area $\mathrm{j}$ is obtained from the SIQR epidemic model:

$$
D_{j}^{k}(t)=p^{k} S_{j}\left(t^{-}\right)
$$

\section{Constraint 2: reserve constraints}

Considering that the vaccine and drug production speed in different periods is not immutable and frozen, and the different production speed will directly affect the amount of drug storage repository. At the time the epidemic begins to spread, drug production speed is very slow. The number of reserve drugs cannot keep up the demand of the people; however, to retard the epidemic spreading, it will get the widespread attention of the government and the international community, so drug production will reach the peak. As a result, the amount of storage reserves will also increase and maintain a certain range.

The law is in accord with the population growth Logistic model. In this model, the natural population growth rate will be affected by the population limit. Here, the storage quantity of each reserve is limited by drug production speed. Therefore, we may think of drug storage repository that follows a Logistic function, and establish the following Logistic growth model:

$$
C A P_{i}=\frac{N}{1+e^{-k}}
$$

In addition, we should ensure that the reserves distribution quantity of the reserve base is not larger than the pool and the drug or vaccine distribution by a plurality of reserve allocation, so we get the following constraints:

$$
\sum_{j=1}^{m} x_{i j}{ }^{k}(t) \leq C A P_{i} \leq D M_{j}^{k}(t)
$$

\section{Constraint 3: decision variables in feasible region}

Because the number of each transport vaccine or drug should be greater than 0 , we can get the third constraint condition as follows:

$$
x_{i j}{ }^{k}(t) \geq 0
$$

If the amount of vaccine distributed to the affected area $j$ that cannot be satisfied, $D M_{j}^{k+1}(t)$ will be regulated.

Accordingly, $D M_{j}^{k+1}(t)$ is given by:

$$
\left\{\begin{array}{l}
\Delta D_{j}^{k}(t)=D_{j}^{k}(t)-\sum_{i=1}^{n} x_{i j}^{k}(t) \\
D M_{j}^{k+1}=D M_{j}^{k+1}(t)-\Delta D_{j}^{k}(t)
\end{array} k=1,2, \ldots, t=k \tau\right.
$$

Where $D M_{j}^{1}(t)=D_{j}^{1}(t)$

\section{Results of the model}


Through a specific example, this section proves the validity of the model and algorithm proposed in this paper when the Ebola breaks out. We choose Sierra Leone in West Africa where the Ebola virus most seriously spreads as an example-7 distribution stations to deliver vaccine or drug to around 13 areas. The original data of infectious disease in early outbreak stage are shown in the Table.

Table 4. Primary parameters about vaccine

\begin{tabular}{|c|c|c|c|c|c|c|c|c|}
\hline \multicolumn{2}{|c|}{ Distribution Station } & I & II & III & IV & V & VI & VII \\
\hline \multirow{2}{*}{ Storage } & 1-th & 480 & 490 & 510 & 470 & 520 & 540 & 500 \\
\hline & 2-th & 490 & 500 & 525 & 485 & 530 & 560 & 520 \\
\hline
\end{tabular}

Table 5. Primary parameters about drug

\begin{tabular}{|c|c|c|c|c|c|c|c|c|}
\hline \multicolumn{2}{|c|}{ Distribution Station } & I & II & III & IV & V & VI & VII \\
\hline \multirow{2}{*}{ Storage } & 1-th & 120 & 110 & 130 & 90 & 140 & 100 & 120 \\
\hline & 2-th & 135 & 120 & 140 & 115 & 150 & 115 & 135 \\
\hline
\end{tabular}

We can get distance between $i$ to $j$ on Google ${ }^{[6]}$.

Table 6. Distance

\begin{tabular}{llllllll}
\hline \multirow{2}{*}{ Affected Area } & \multicolumn{7}{c}{ Distance } \\
& I & II & III & IV & V & VI & VII \\
\hline 1 & 20 & 185 & 239 & 194 & 210 & 69 & 106 \\
2 & 185 & 32 & 422 & 367 & 401 & 117 & 289 \\
3 & 239 & 422 & 24 & 185 & 119 & 305 & 118 \\
4 & 194 & 367 & 185 & 23 & 110 & 253 & 186 \\
5 & 210 & 401 & 119 & 110 & 31 & 276 & 127 \\
6 & 113 & 296 & 246 & 269 & 250 & 180 & 128 \\
7 & 263 & 454 & 173 & 147 & 50 & 330 & 181 \\
8 & 69 & 117 & 305 & 253 & 276 & 19 & 173 \\
9 & 246 & 419 & 302 & 133 & 212 & 306 & 238 \\
10 & 191 & 167 & 340 & 239 & 273 & 132 & 273 \\
11 & 106 & 289 & 118 & 186 & 127 & 173 & 32 \\
12 & 76 & 227 & 312 & 267 & 283 & 110 & 179 \\
13 & 107 & 279 & 208 & 110 & 144 & 166 & 143 \\
\hline
\end{tabular}

Table 7. Amount of vaccine demanded

\begin{tabular}{llllllllllllll}
\hline Area & 1 & 2 & 3 & 4 & 5 & 6 & 7 & 8 & 9 & 10 & 11 & 12 & 13 \\
\hline 1 -th & 315 & 945 & 6 & 420 & 187 & 525 & 105 & 180 & 210 & 1200 & 45 & 420 & 365 \\
2-th & 275 & 894 & 4 & 395 & 213 & 476 & 121 & 162 & 179 & 1188 & 43 & 367 & 343 \\
\hline
\end{tabular}

Table 8. Amount of drug demanded

\begin{tabular}{llllllllllllll}
\hline Area & 1 & 2 & 3 & 4 & 5 & 6 & 7 & 8 & 9 & 10 & 11 & 12 & 13 \\
\hline 1 -th & 47 & 146 & 1 & 85 & 22 & 75 & 16 & 35 & 30 & 154 & 5 & 65 & 35 \\
2-th & 43 & 127 & 0 & 91 & 23 & 54 & 13 & 41 & 27 & 136 & 4 & 53 & 37 \\
\hline
\end{tabular}


Table 9. Amount of vaccine distributed

\begin{tabular}{llllllllllllll}
\hline Area & 1 & 2 & 3 & 4 & 5 & 6 & 7 & 8 & 9 & 10 & 11 & 12 & 13 \\
\hline 1-th & 278 & 945 & 6 & 420 & 187 & 502 & 98 & 180 & 210 & 980 & 45 & 420 & 365 \\
2-th & 275 & 894 & 4 & 395 & 203 & 476 & 87 & 162 & 179 & 1030 & 43 & 352 & 343 \\
\hline
\end{tabular}

Table 10. Amount of drug distributed

\begin{tabular}{llllllllllllll}
\hline Area & 1 & 2 & 3 & 4 & 5 & 6 & 7 & 8 & 9 & 10 & 11 & 12 & 13 \\
\hline 1 -th & 47 & 146 & 1 & 73 & 22 & 64 & 16 & 35 & 30 & 132 & 5 & 65 & 35 \\
2-th & 36 & 127 & 2 & 91 & 23 & 54 & 6 & 34 & 27 & 136 & 4 & 53 & 37 \\
\hline
\end{tabular}

According to the initial data and parameter setting of the above model, we will carry on the simulation analysis of the dynamic model of emergency material distribution based on epidemic spreading rules. We assume that the periodic pulse vaccination is for 5 days, every 0.15 increases in the vaccination rate. We can obtain the numerical solution of infected diffusion model SIQR (1) by ODE45 function in MATLAB, as shown in Figure 3. From Figure 3, we can see that with the elapse of time, the number of people infected is nearly 0 , and spreading in infectious diseases has been effectively controlled. We used two pulses as the research object, the first two pulses vaccine and drug demand forecasting inoculated respectively, as shown in Table 7 and Table 8.

Based on the SIQR epidemic model solving and epidemic clustering, the dynamic distribution model 3 is solved by using Lingo, and the calculation results of actual transport numbers are shown in the Table 9 and 10.

Comparing Table 7 with Table 9, in the first pulse, it is not difficult to determine that the amount of demanded vaccine which is associated with areas 1, 3, 6, 7 and 10 is not satisfied. When it can be learned from Table 3 that areas 1, 3, 6 and 7 are all listed in group 2 and group 3, the urgency degrees of which are both lower than that of group 1. In the second pulse, the unsatisfied areas 3, 5, 7, 12 are all included in group 2 and group 3. Due to the large demand associated with area 10 , a fraction of vaccine is carried to area 10 .

Comparing Table 8 with Table 10, in the first pulse, it is not difficult to determine that the amount of drug which is demanded associated with areas 4, 6 and 10 is not satisfied while it can be learned from Table 3 that they are all listed in group 2 and group 3. In the second pulse, the unsatisfied areas 1, 7, 8, 11 are all included in group 3. Due to the large demand associated with area 10 , a fraction of drug is carried to area 10.

As a consequence, we can draw the conclusion that the proposed approach meets the objective to effectively control the affected areas with a relatively high degree of urgency when the amount of medicine is deficient.

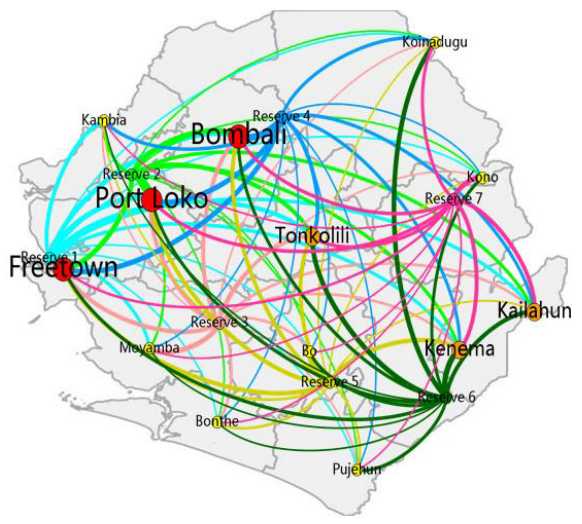

Figure 4. Transportation system of Sierra Leone 


\section{Conclusions}

With the rapid spread of the Ebola virus in West Africa, it is of great significance to optimize the eradication of Ebola or at least its current strain. This paper presents a clustering method to establish a series of dynamic vaccine distribution operations for quickly responding to urgent medicinal needs.

Based on the SIQR epidemic model with pulse vaccination, the amount of vaccine demanded is forecasted. By the cluster analysis, the affected areas are clustered and the priority is determined. The Dynamic Medical Material Delivery model is formulated based on the two aforementioned mechanisms with the goal of reducing the transportation cost and decreasing the unsatisfied demand. The results indicate that the proposed approach helps to control the affected areas with a relatively high degree of urgency. Nonetheless, there is still great potential for improving the performance of the distribution plan.

Furthermore, more influential factors can be integrated into the attributes for advancing the accuracy of the priorities associated with a given affected area. Overall, the clustering approach can be employed by the decision-makers to efficiently classify the affected areas based on different degrees of urgency and make corresponding decisions scientifically.

\section{References}

1. Kermack M \& Mckendrick A. 1927. Contributions to the mathematical theory of epidemics. Part I. Proc. Soc. A, 115: 700-721.

2. Dai Dongfu \& Zhao Lindu. 2008. Biological hazard diffusion dynamic research based on SIQR model under a planned pulse vaccination strategy. Highlights of Science Paper Online, 1(12): 1315-1321.

3. Xu Jingjing \& Wang Haiyan. 2010. Dynamic vaccine distribution model based on epidemic diffusion rule and clustering approach. Journal of Southeast University, 5.

4. Number of Ebola Cases and Deaths. [2015-2-7]. https://data.hdx.rwlabs.org/dataset/ebola-cases-2014

5. Ebola Virus Disease Distribution Map. [2015-2-7]. http://www.cdc.gov/vhf/ebola/outbreaks/history/distribution-map.html.

6. Ditance.[2015-2-7].https://data.hdx.rwlabs.org/dataset/travel-distance-and-time-chart.

7. Ebola. Wikipedia.[2015-2-7]http://en.wikipedia.org/wiki/Ebola_virus_disease 